\title{
La gouvernance des fonds souverains et le nationalisme économique
}

\author{
Gilles Saint-Paul \\ Toulouse School of Economics \\ Birkbeck College
}

Dans cet article, on s'interroge sur le comportement des fonds souverains ainsi que la mesure dans laquelle leurs objectifs diffèreraient de ceux d'agents purement privés. Nous nous plaçons résolument dans une perspective positive : plutôt que de prescrire une stratégie "optimale " pour ces fonds, nous nous demandons plutôt quelle sera la stratégie qu'ils adopteront. Comme on le verra, cela dépend des hypothèses que l'on fait sur leur nature.

Une première hypothèse serait que les fonds souverains ne sont pas différents des autres intermédiaires financiers et que comme eux ils maximisent le rendement de leur capital. Dans ce cas les fonds souverains n'auront pas d'effets allocatifs si ce n'est peut-être qu'ils représentent une forme d'épargne forcée, dans la mesure où à la place d'un fonds souverain l'Etat aurait pu rembourser la mise initiale aux citoyens du pays considéré. Même s'il y a épargne forcée, les agents privés peuvent annuler ses effets en désépargnant une quantité égale au capital du fonds souverain ; c'est ce qui se passerait en l'absence d'imperfections sur les marchés financiers par le simple jeu de la prise en compte des dividendes futurs du fond souverain - et ceux-ci peuvent être explicites ou implicites à travers des impôts plus faibles - dans la décision de consommation courante des agents.

C'est donc dans la mesure où leur objectif diffère de celui des autres intermédiaires que les fonds souverains auront des effets réels. Ceci peut-être le cas pour deux raisons :

-d'une part, le fonds souverain peut faire face à moins de contraintes que les autres fonds. Par exemple, d'après certains auteurs, il n'est pas soumis au risque de liquidité (c'est-à-dire d'un retrait brutal des investisseurs) et donc peut se permettre d'investir dans des actifs eux-mêmes moins liquides en obtenant en contrepartie un rendement plus élevé. Notons qu'il n'est pas clair qu'un fond souverain n'ait pas de risque de liquidité. Une partie du passif de la caisse des dépôts (si l'on veut bien la considérer comme un fonds souverain), par exemple, consiste en des livrets de caisse d'épargne qui sont parfaitement liquides et vulnérables à un " run " tout comme les dépôts d'une banque commerciale. De plus, il existe un risque de liquidité en provenance de l'Etat lui-même, qui peut être tenté de puiser dans les actifs du fonds souverains pour faire face à des besoins de financement exceptionnels. Qu'on se souvienne, par exemple, du transfert forcé entre l'Etat et le fonds de pension de France Télécom. Il est cependant vrai que si une panique peut être autoréalisatrice, chaque épargnant retirant ses actifs pour l'unique raison que les autres en font de même, cela n'est pas le cas avec le risque de liquidité de l'Etat.

-d'autre part - et c'est ce qui nous intéresse en premier lieu ici - le fonds souverain peut avoir des objectifs intrinsèquement différents de ceux d'un intermédiaire financier normal. On peut considérer deux possibilités. Tout d'abord, on peut supposer que le fonds souverain a un comportement " altruiste " et maximise le bien-être total des citoyens du pays. C'est ce qui aurait lieu s'il était géré par un planificateur bénévole qui prendrait en compte l'intérêt de tous les citoyens. Ensuite, on peut 
supposer que le fonds souverain est géré par des politiciens et se conforme à leurs intérêts personnels. Cela peut signifier qu'il maximise leur probabilité de réélection, et on a alors affaire à un fonds "opportuniste ", ou alors qu'il maximise le bien-être de l'électorat des partis de la coalition au pouvoir, à l'exclusion des autres, et il s'agit alors d'un fonds " partisan " (ces deux aspects ne sont d'ailleurs pas mutuellement exclusifs, mais il est utile de les séparer).

Ceci étant posé, la question est de savoir dans quelle mesure les choix d'investissements des fonds souverains diffèreraient des choix des investisseurs privés. Dans ce qui suit, nous analysons différents mécanismes ainsi que leur pertinence suivant la nature du fonds souverain considéré.

\section{La prise en compte d'externalités}

Un premier effet possible est la prise en compte d'externalités dans les choix d'investissement. Considérons par exemple le choix entre deux investissements $A$ et $B$. Supposons que $A$ ait un taux de rendement plus élevé mais utilise une technologie polluante qui exerce des effets négatifs sur certains citoyens. On peut supposer qu'un fonds privé sera uniquement concerné par la maximisation du taux de rendement et choisira la technologie A. Inversement, un fonds souverain altruiste ne maximise pas le taux de rendement mais le bien être des résidants ; si ce fonds souverain représente le pays où l'externalité s'exerce, alors il pourra préférer l'investissement « propre » $B$.

Ce raisonnement apparemment simple nécessite cependant un raffinement. En effet, on pourrait objecter qu'un fonds privé dont les actionnaires sont soumis à l'externalité aurait également intérêt à choisir le projet $B$. En effet, en dernière analyse ce n'est pas le taux de rendement que maximise une entreprise capitaliste mais le bien-être de ses actionnaires. Mais un tel fonds privé a peu de chances d'être stable : en effet chaque actionnaire a intérêt à revendre individuellement ses parts à un investisseur " étranger » représentants des épargnants non affectés par l'externalité, et qui ayant pris le contrôle de l'entreprise choisira alors le projet A plutôt que le projet B. Dans la mesure où la majorité des actionnaires revendent leurs part, il est optimal pour chaque actionnaire individuel de le faire puisque le fonds " étranger " est prêt à payer plus cher pour l'action que sa valeur initiale (reflétant ainsi les gains monétaires de choisir le projet A plutôt que B) et que sa propre décision de vendre ses parts est sans effet sur le contrôle de l'entreprise. C'est là le problème bien connu du "free rider " des petits actionnaires. ${ }^{1}$ En revanche, un fonds souverain n'est évidemment pas vulnérable à une telle possibilité de prise de contrôle.

L'existence d'externalités est-elle un argument justifiant l'existence de fonds souverains ? Rien n'est moins sûr. Indépendamment du fait que le fonds souverain n'est pas nécessairement altruiste, on sait qu'une externalité se corrige par une taxe pigovienne appropriée ; si par exemple il existe une taxe sur les émissions polluantes, celle-ci réduira le rendement net du projet $A$ d'une quantité représentant exactement le coût externe associé à ce projet. Dans ce cas la comparaison des taux de rendements des deux projets est à nouveau le bon critère du point de vue du bien-être social et une entreprise capitaliste choisira celui qui est socialement optimal. En d'autres termes à partir du moment où le gouvernement met en place les instruments adéquats il n'y a pas lieu d'une intervention supplémentaire à travers un fonds souverain.

\footnotetext{
${ }^{1}$ Voir par exemple J. Tirole, The theory of corporate finance, Princeton University Press, 2006
} 


\section{L'effet de transfert}

Par " effet de transfert " j'entends le fait qu'un fonds souverain altruiste prendra en compte les transferts implicites entre les consommateurs résidants et le reste du monde dans ses choix $d^{\prime}$ 'investissement internationaux, tandis qu'un fonds capitaliste ne considèrera que les profits. Cela peut s'illustrer tout particulièrement dans un contexte de concurrence imparfaite. Considérons par exemple, suivant en cela les travaux de Neven et Seabright ${ }^{2}$, le cas $d^{\prime} u n$ monopole étranger (Boeing) ; il est possible de créer, moyennant un certain coût d'investissement, une autre entreprise domestique dans le même secteur (Airbus), le transformant ainsi en duopole. Cela a deux effets principaux. D'une part, le prix du produit baissera sous l'effet de la concurrence accrue; au lieu d'être facturé au prix de monopole, celui-ci sera facturé à un prix de duopole qui lui est inférieur. II en résulte une hausse du surplus du consommateur dans le pays et à l'étranger, ainsi qu'une baisse globale des profits. On sait également que le bien-être total augmente, c'est-à-dire que la hausse du surplus des consommateurs est supérieure à la baisse des profits. D'autre part, les profits du secteur ne sont plus entièrement appropriés par les résidants étrangers; une partie est appropriée par les actionnaires de la nouvelle entreprise domestique.

Au moment du choix de l'investissement, un investisseur capitaliste ne considérera donc que les profits. Ceux-ci seront d'autant plus faibles que l'effet du duopole sur la concurrence est fort. Le fonds souverain altruiste, au contraire, considérera que le rendement de l'investissement est égal à la somme des profits de la nouvelle entreprise et du gain pour les consommateurs domestiques de la concurrence accrue ; cette dernière composante est d'autant plus grande que la baisse des prix est élevée. Comme le gain total pour le fonds souverain est supérieur au profit, il aura plus souvent tendance à investir qu'un fonds purement capitaliste.

L'investissement crée un transfert de profits de Boeing vers Airbus que ce dernier prend en compte qu'il soit public ou privé. Mais il crée aussi un transfert de Boeing vers le consommateur européen (i.e. les compagnies aériennes) que prendrait en compte un fonds souverain altruiste mais pas un investisseur privé.

Enfin, on peut également considérer l'effet sur le bien-être mondial de l'investissement. Une partie de ce bien-être n'est prise en compte ni par un fonds capitaliste ni par un fonds souverain domestique : il s'agit de la hausse du surplus des consommateurs étrangers associée à la baisse des prix. Une autre partie est prise en compte par les fonds domestiques mais ne change rien au bienêtre mondial: la fraction des profits totaux transférés de l'étranger vers les actionnaires de la nouvelle entreprise. Comme ces deux effets s'opposent, on peut avoir des cas où l'investissement a lieu alors qu'il réduit le bien-être mondial, et des cas où il n'a pas lieu alors qu'il l'augmenterait .

Il est utile d'illustrer cette discussion à l'aide d'un exemple numérique. Le tableau ci-dessous représente dans sa colonne 1 le surplus des différents acteurs dans un cas de figure où le prix de monopole est $50 \%$ supérieur au coût de production, tandis que la colonne 2 nous donne les

\footnotetext{
${ }^{2}$ Damien Neven and Paul Seabright (1995), « European Industrial Policy : The Airbus Case », Economic Policy, 21, 313-58
} 
grandeurs corresponda ntes dans le cas où le prix de duopole ne lui est supérieur que de $10 \%{ }^{3}$ On voit que le profit d'Airbus est égal à 2, le gain pour les résidants européens de $13-5=8$, le gain pour les résidants américains de $13-15=-2$, et le gain pour l'économie mondiale de $26-20=6$. Dans cette configuration, un fonds privé investira si le coût d'investissement est inférieur ou égal à 2 , tandis que le fonds souverain investira dès lors qu'il est inférieur à 8 . Mais s'il est supérieur à 6 cela réduit le bien-être mondial. De fait le gouvernement américain est prêt à payer 2 à l'Europe pour qu'elle s'abstienne de créer Airbus, et si le coût d'investissement est supérieur à 6 il est optimal pour elle d'accepter ce "pot-de-vin " plutôt que de mettre en route le projet. La troisième colonne nous donne les distributions des gains et des pertes dans le cas où il y aurait forte collusion au sein du duopole et où le prix resterait $40 \%$ supérieur au coût de production. Dans ce cas les profits d'Airbus sont égaux à 5 , le gain pour le consommateur Européen à 7 et celui pour le consommateur mondial seulement à 2. Dans le cas où le coût d'investissement est compris entre 2 et 5 , l'investissement est profitable même pour un fonds privé mais il réduit néanmoins le bien-être mondial.

\begin{tabular}{|l|l|l|l|}
\hline & $\begin{array}{l}\text { Boeing } \\
\text { seul, } \\
\mathrm{p}=\mathrm{c}^{*} 1.5\end{array}$ & $\mathrm{P}=\mathrm{c}^{*} 1.1$ & $\mathrm{P}=\mathrm{c}^{*} 1.4$ \\
\hline $\begin{array}{l}\text { Consommateur } \\
\text { américain }\end{array}$ & 5 & 11 & 6 \\
\hline $\begin{array}{l}\text { Consommateur } \\
\text { européen }\end{array}$ & 5 & 11 & 6 \\
\hline $\begin{array}{l}\text { Entreprises } \\
\text { américaines }\end{array}$ & 10 & 2 & 5 \\
\hline $\begin{array}{l}\text { Entreprises } \\
\text { européennes }\end{array}$ & 0 & 2 & 5 \\
\hline $\begin{array}{l}\text { Résidants } \\
\text { américains }\end{array}$ & 15 & 13 & 11 \\
\hline $\begin{array}{l}\text { Résidants } \\
\text { Européens }\end{array}$ & 5 & 13 & 22 \\
\hline & 20 & 26 & 11 \\
\hline Monde & & & \\
\hline
\end{tabular}

Tableau 1 - Les transfers internationaux associés à une entrée sur le marché.

\footnotetext{
${ }^{3}$ II s'agit d'un modèle avec une demande qui décroît exponentiellement avec le prix. On a supposé que les économies américaines et européennes étaient de même taille et que la part de marché de chaque entreprise dans le cas du duopole est de $50 \%$.
} 
Dans tous les cas de figure du Tableau 1, on a supposé que le consommateur européen représente la moitié de la demande mondiale, l'autre moitié provenant des consommateurs américains. Le tableau 2 décrit une situation où les consommateurs américains représentent $75 \%$ du marché (mais la part de marché d'Airbus reste de $50 \%$, comme dans le tableau 1). Ils bénéficient alors bien plus de la baisse des prix que le consommateur européen, et on trouve que les gains mondiaux sont supérieurs aux gains pour les résidants européens, malgré les profits transférés vers l'Europe. Le gain mondial est de 6.6, alors que le gain pour l'Europe n'est que de 5.3. Si le coût de l'investissement est entre ces deux valeurs, le fonds souverain n'investira pas (et un fonds capitaliste encore moins) alors que ce serait souhaitable pour le bien-être mondial - et, à sa place, un fonds « mondial » investirait.

Même si, comme on l'a vu plus haut, un fonds capitaliste peut être amené à prendre la bonne décision du point de vue du bien-être mondial dans une situation où un fonds souverain altruiste ne le ferait pas, cela ne peut évidemment pas être le cas si l'on se limite au bien-être des résidants puisque celui-ci définit précisément l'objectif du fonds souverain.

Cependant, cela suppose qu'un tel comportement altruiste est effectivement à la portée du fonds, or cela n'est plus évident dès lors qu'on considère les problèmes de gouvernance des fonds souverains ainsi que sa vulnérabilité aux critères politiques.

\section{Les problèmes de gouvernance}

Un fonds privé est soumis à une discipline stricte par les marchés: dès lors que son taux de rendement est inférieur à la performance du marché, les investisseurs peuvent s'en retirer et placer leur argent ailleurs ; cette concurrence par la réallocation de l'épargne force le fonds à se limiter aux investissements les plus productifs de façon à se maintenir sur la frontière d'efficacité entre le risque et le rendement, une baisse du rendement n'étant acceptable que si elle est accompagnée d'une baisse concomitante du risque.

En ce qui concerne le fonds souverain, d'une part il n'est pas exposé à cette concurrence puisqu'il repose sur une épargne captive ; d'autre part il n'existe pas de mesure objective de sa performance susceptible de faire l'unanimité, puisqu'il ne maximise pas le rendement mais une notion d' " intérêt national » bien plus complexe à appréhender. Ces deux facteurs accroissent les opportunités pour les dirigeants de ces fonds, ainsi que pour les dirigeants des entreprises dont ils possèdent des participations, d'extraire des rentes à leur profit plutôt que de choisir les investissements les plus profitables. Certes, une entreprise contrôlée par des actionnaires privés peut artificiellement augmenter son taux de rendement à l'aide de pratiques comptables douteuses ou en compromettant le long terme (par exemple en liquidant des actifs intangibles tels que la réputation). Mais ces manipulations ne peuvent fonctionner que si elles font augmenter la rentabilité mesurée du capital, tandis qu'une entreprise dont l'objectif n'est plus la rentabilité du capital peut également générer des rentes privées à l'aide de projets peu efficaces, ce qui est évidemment beaucoup plus facile. En théorie, le conseil d'administration d'un fonds souverain devrait évaluer sa performance sur la base du surplus social total généré par ses projets, ce qui requiert des outils économétriques sophistiqués et est sujet à des erreurs de mesure considérables, une telle évaluation devant nécessairement reposer sur un modèle dont la spécification et l'estimation comportent une grande part d'arbitraire. 
En pratique, on s'attend à ce que le fonds prétende que ses investissements sont bénéfiques pour la collectivité de façon plutôt vague et qu'il soit difficile de distinguer de vraies contributions sociales de la distribution de rentes privées. Ainsi, Kramarz et Thesmar (2006) ont étudié la performance des P.D-G issus des corps de hauts fonctionnaires qui recrutent à la sortie de l'ENA et de l'Ecole Polytechnique et montrent que leur emploi est largement à l'abri des baisses de la rentabilité de leurs entreprises, tandis que pour le dirigeant moyen la probabilité de perte d'emploi augmente de $0.67 \%$ pour chaque point de réduction du taux de rentabilité. Ils montrent également que le fait pour ces dirigeants d'être membres de multiples conseils d'administration a un impact négatif sur la valeur des entreprises ${ }^{4}$. Leur interprétation est qu'il y a là des réseaux qui confisquent la démocratie actionnariale au détriment du profit, tout en extrayant des rentes pour leurs membres. Mais si les entreprises considérées ont un rôle " social ", on peut fort bien objecter que le rendement financier n'est plus un objectif valable et que ces réseaux, de par leur proximité avec le politique, jouent un rôle positif de courroie de transmission entre les choix collectifs et les conseils d'administration telle était d'ailleurs la défense de Jean-Yves Haberer lors du scandale du Crédit Lyonnais : il aurait travaillé en étroite collaboration avec les politiques pour financer des projets qui, quoique peu rentables, préservaient des emplois dans l'industrie française en permettant aux entreprises en difficulté d'accéder au crédit. L'inamovibilité relative des membres du réseau face à la baisse de la valeur de l'entreprise n'est alors plus l'indication d'une rente de situation mais simplement le signe que cet mesure n'est pas pertinente.

\section{Les biais politiques}

Intuitivement, on pourrait s'attendre à ce que les problèmes de gouvernance que nous venons de décrire soient exacerbés si le fonds souverain n'est pas "altruiste " mais poursuit des objectifs électoraux. Paradoxalement, il y a des raisons de penser que ce n'est pas le cas. En effet, ils sont à nouveau dans une logique de "marché ", le marché pertinent étant cependant électoral plutôt qu'économique, et cela permet de réintroduire des mesures objectives de leur performance ainsi qu'un certain degré de concurrence. Par exemple, si un projet dans une région donnée ne se traduit pas par une amélioration des sondages en faveur du gouvernement dans cette région, on peut en conclure que le rendement politique du fonds est faible et envisager de remplacer ses dirigeants. II est donc paradoxalement plus difficile pour ces derniers d'extraire des rentes privées que dans le cas d'un fonds altruiste. Bien évidemment, cet avantage est compensé par le fait qu'un fonds opportuniste ou partisan ne maximise plus le bien-être de l'ensemble des citoyens mais poursuit des objectifs électoraux. On notera cependant qu'il existe une corrélation positive entre les deux types d'objectifs puisque pour remporter des élections un décideur doit poursuivre une politique qui bénéficie à suffisamment de personnes. Cependant, cet effet et sans doute faible et il n'est pas difficile d'énumerer les inefficacités inhérentes à un fonds non altruiste.

Par exemple, un fonds souverain opportuniste verra ses objectifs rythmés par les échéances électorales et sera donc vulnérable au court-termisme. On peut imaginer qu'à l'approche d'une élection, un tel fonds soit biaisé en faveur de projets qui génèrent du cash-flow rapidement en dépit de leur faible rentabilité de long terme, ou qu'il investisse dans des régions ou des secteurs

\footnotetext{
${ }^{4}$ Francis Kramarz et David Thesmar, « Social networks in the boardroom », CEPR Discussion Paper No. 5496
} 
électoralement pivotaux alors qu'il existerait des alternatives supérieures du point de vue du bienêtre social.

Un fonds souverain partisan sera biaisé en faveur de projets dont les retombées sont positives pour les électeurs du parti, au détriment des autres électeurs. Le transfert entre les deux groupes peut se faire à travers la faible rentabilité du projet, ce qui équivaut indirectement à un impôt sur les revenus du capital ; mais d'autres mécanismes jouent également, à travers l'impact du projet sur les prix, les salaires, ou la localisation de l'activité économique. Ainsi, un fonds altruiste ne favoriserait un pôle de développement local que dans la mesure où les effets exercés sur ce pôle sur les régions limitrophes sont positifs (par exemple à travers la demande accrue adressée à ces régions, ou l'effet des nouvelles infrastructures de transport sur leur productivité), tandis qu'un fonds partisan le favoriserait même si ces effets étaient négatifs (par exemple à travers la migration des travailleurs qualifiés ou des entreprises, ou encore une hausse de la rente foncière), dans la mesure où la région bénéficiaire est surreprésentée dans l'électorat du parti au pouvoir.

\section{Pourquoi cet outil ?}

Nous avons analysé un certain nombre de raisons pour lesquelles les fonds souverains se comporteraient différemment des fonds privé. Mais les objectifs que le politique pourrait se fixer à travers la stratégie d'un fonds souverain pourraient tout aussi bien être atteints à l'aide d'autres politiques telles que subventions, impôts, réglementations, etc. Si ces politiques sont fixées de façon optimale alors le gouvernement n'a pas besoin d'un fonds souverain et si pour une raison quelconque il en existait un il lui imposerait les mêmes objectifs de rentabilité qu'à un fonds privé ; en effet l'arsenal de mesures correctives prises par ailleurs garantit que les signaux du marché sont corrects du point de vue de l'objectif du gouvernement. L'analyse qui précède se rapporte donc au comportement d'un fonds souverain en l'absence d'un éventail suffisamment large d'instruments de politique économique à la disposition du gouvernement.

Il est intéressant de noter que cet éventail peut être restreint précisément à cause de traités limitant la marge de manœuvre de l'Etat; on peut penser à l'Union Européenne et à l'OMC. II n'est pas possible pour un Etat membre de l'UE de discriminer contre les résidants des autres pays membres. Dans le cadre de la discussion ci-dessus sur l'effet de transfert, cela limite évidemment les possibilités d'extraire des rentes des résidants étrangers ${ }^{5}$. Par exemple, la France ne peut pas maintenir un monopole d'Etat dans un secteur tel que le ferroviaire ou l'électricité tout en bénéficiant de l'ouverture à la concurrence dans les pays voisins. C'est pourtant ce qu'elle serait tentée de faire si elle désire extraire une partie des rentes dans ces pays (à supposer qu'elles ne soient pas entièrement éliminées par la concurrence) tout en empêchant les étrangers de s'approprier celles qui existent en France ${ }^{6}$. C'est bien parce que la lutte pour ces rentes est un jeu à somme nulle qu'il

\footnotetext{
${ }^{5}$ Pour une discussion du nationalisme économique dans un contexte européen, voir EEAG, The EEAG report on the European Economy, 2007, ch. 6, Munich : CESIfo.

${ }^{6} C^{\prime}$ est d'ailleurs ce qui s'est passé lors de l'acquisition par EdF de parts dans Montedison en 2001 tandis que la réciproque n'était pas possible du fait qu'EdF restait un monopole d'Etat, d'où les protestations des Italiens à l'époque.
} 
existe des accords internationaux qui harmonisent les barrières douanières (dans le cas de l'OMC) et les règles concurrentielles (dans le cas de l'UE).

Mais, bien qu'il soit dans l'intérêt des Etats de souscrire à ces règles, ils n'en sont pas moins tentés de les contourner pour leur propre compte. Le fonds souverain peut alors être considéré comme une façon détournée de les transgresser sans pour autant mettre en place des règles locales explicitement discriminatoires. Le gouvernement français ne peut pas imposer un taux de TVA différent sur les automobiles selon leur origine mais un fonds souverain français peut bien plus facilement investir dans une entreprise française plutôt qu'étrangère ${ }^{7}$. Même si le fonds est altruiste il ne prend pas en compte le bien-être des étrangers et cela ouvre la possibilité d'accords coopératifs multilatéraux qui restreindraient le comportement de ces fonds de la même manière que les accords existants de l'OME et de l'UE restreignent celui des gouvernements. En d'autres termes, on peut craindre que les fonds souverains ne soient un nouveau vecteur du nationalisme économique qui réduise le degré de coopération internationale.

On peut également mentionner le cas où un fonds souverain serait utilisé stratégiquement dans le cadre d'un conflit pour affaiblir l'adversaire ; c'est un cas extrême de l'effet de transfert où le jeu peut être à somme négative, c'est-à-dire que les choix du fonds souverain sont également mauvais économiquement du point de vue domestique mais favorisent un règlement du conflit dans le sens souhaité. Un exemple qui vient à l'esprit serait l'acquisition stratégique de la dette publique d'un pays tiers dans le but d'avoir l'option de déstabiliser sa monnaie en la revendant massivement. Cela n'est clairement pas dans l'intérêt du vendeur puisque la valeur de l'actif qu'il détient en grande quantité baisse, mais le coût imposé à l'adversaire par la déstabilisation monétaire peut être encore plus élevé et l'amener à des concessions rapides ${ }^{8}$.

\section{Conclusion}

Les importants fonds souverains que nous connaissons actuellement sont le résultat à la fois des déséquilibres mondiaux de balances des paiements et de la forte implication de l'Etat dans les économies de la Chine et des pays producteurs d'hydrocarbures. Dans le cas de pays Européens, il n'existe pas de surplus de balance des paiements permettant de créer des fonds de cette taille. La France semble penser qu'un tel fonds est important comme vecteur d'une stratégie industrielle. Audelà de la désirabilité de telles stratégies, il ressort de notre discussion qu'il n'est pas clair qu'un

\footnotetext{
${ }^{7}$ Cependant, une telle politique est sans doute sujette à un recours auprès de la commission européenne en tant qu'aide déguisée. Mais il est indéniable qu'un tel recours a moins de chances d'aboutir que dans le cas d'une subvention explicite.

${ }^{8}$ L'affaire de Suez (1956) obéit à un scénario légèrement différent. Les Etats-Unis n'ont sans doute pas été proactifs dans la spéculation contre la livre Sterling mais ils ont refusé d'intervenir sur les marchés des changes pour soutenir la livre et ont bloqué l'accès de la Grande-Bretagne au crédit du FMI ainsi que son utilisation d'actifs américains comme collatéral pour les emprunts commerciaux. Voir Adam Klug et Gregor W. Smith, "Suez and Sterling, 1956", Explorations in Economic History, 36, 3, Juillet 1999, 181-203.
} 
fonds souverain soit l'outil adéquat; par ailleurs, cela procède d'une résurgence du nationalisme économique qui risque de remettre en question les acquis de la coopération internationale. 